\title{
Primary intracranial neuroendocrine tumor with ectopic adrenocorticotropic hormone syndrome: A rare and complicated case report and literature review
}

\author{
HAILONG LIU, MINGSHAN ZHANG, XUAN WANG, YANMING QU, HONGWEI ZHANG and CHUNJIANG YU \\ Department of Neurosurgery, Sanbo Brain Hospital, Capital Medical University, Beijing 100093, P.R. China
}

Received October 2, 2015; Accepted March 16, 2016

DOI: $10.3892 / \operatorname{mco} .2016 .904$

\begin{abstract}
Neuroendocrine tumors (NETs) and ectopic adrenocorticotropic hormone $(\mathrm{ACTH})$ syndrome are frequent in adult patients. However, primary intracranial NETs, exhibiting immunonegativity for ACTH, high serum ACTH level and treated with anterior skull base reconstruction, are rare and complicated. We herein present a case of a primary intracranial NET immunonegative for ACTH, resulting in ectopic ACTH syndrome. A 40-year-old woman presented with intermittent rhinorrhea, rapid weight gain, polydipsia, polyuria, hypertension, dimness, bilateral exophthalmus, diminution of vision in the left eye and pigmentation of the skin of the face and trunk. Computed tomography (CT) and magnetic resonance imaging scans revealed a sizeable enhancing tumor in the anterior cranial fossa, which infiltrated the sphenoid and ethmoid sinuses bilaterally, the left maxillary sinus and the nasal cavity. Abdominal CT scans revealed bilateral adrenal hyperplasia. The biochemical findings included hypokalemia and high glucose, cortisol, plasma ACTH, 24-h urinary free cortisol and testosterone levels. The neoplasm was exposed through a right frontal craniotomy, while anterior skull base reconstruction was performed during surgery. The intracranial surgery achieved gross removal of the tumor; however, part of the tumor remained in the nasal cavity. Histopathological examination of the surgical specimen confirmed the diagnosis of a low-grade small-cell NET, exhibiting immunonegativity for ACTH. A postoperative abdominal CT scan demonstrated bilateral regression of the adrenal gland hyperplasia and the serum ACTH level returned to normal after 16 days. To the best of our knowledge, there are no previous reports of primary intracranial NETs, immunohistochemically negative for ACTH, resulting in ectopic ACTH syndrome.
\end{abstract}

Correspondence to: Dr Chunjiang Yu, Department of Neurosurgery, Sanbo Brain Hospital, Capital Medical University, No. 50 Xiangshan South Road, Beijing 100093, P.R. China E-mail: liuhailonger@163.com

Key words: neuroendocrine tumor, ectopic adrenocorticotropic hormone syndrome, anterior skull base reconstruction, diagnosis, treatment

\section{Introduction}

Neuroendocrine tumors (NETs) originate from the diffuse neuroendocrine system, which is composed of cells functioning as neurocytes, as well as endocrine cells. This type of tumor most commonly occurs in the respiratory system and gastrointestinal tract, while intracranial origin is rare $(0.74 \%)$. We herein present a case of a 40 -year-old woman with a primary intracranial NET, which was immunonegative for adrenocorticotropic hormone (ACTH), resulting in ectopic ACTH syndrome.

\section{Case report}

A 40-year-old woman was admitted to Sanbo Brain Hospital (Beijing, China) with a 6-year history of intermittent bilateral rhinorrhea, a 2-month history of rapid weight gain, polydipsia, polyuria and weakness, and a 2-week history of dimness. On examination, the patient was found to have hypertension, dimness, bilateral exophthalmus, diminution of vision in the left eye, pigmentation of the skin of the face and trunk and grade 4 muscle weakness; other findings were unremarkable. Computed tomography (CT) and magnetic resonance imaging scans revealed a sizeable contrast-enhanced tumor in the anterior cranial fossa, which infiltrated the sphenoid and ethmoid sinuses bilaterally, the left maxillary sinus and the nasal cavity (Figs. 1-4). The abdominal CT scans revealed bilateral adrenal hyperplasia. On laboratory biochemical tests, the serum potassium level was $1.92-2.82 \mathrm{mmol} / \mathrm{l}$ and the fasting blood glucose (FBG) level 14.9-20.0 mmol/1. The basal ACTH level was $33.72 \mathrm{pmol} / 1$ (normal, <2.2 pmol/1), the 6 a.m. serum cortisol level was 1,779.78 nmol/1 (normal, 83-359 nmol/1), the 24-h urinary free cortisol level was $\leq 11,194.64 \mathrm{nmol} / 24 \mathrm{~h}$ (normal, 53.2-789.4 nmol/24 h), and the testosterone level was $8.43 \mathrm{nmol} / \mathrm{l}$ (normal, $0.17-2.53 \mathrm{nmol} / \mathrm{l}$ ).

The provisional diagnosis was anterior cranial base tumor with ectopic ACTH syndrome. The neoplasm was exposed intradurally and extradurally through a right frontal craniotomy, while anterior skull base reconstruction was performed during surgery. The intracranial surgery achieved gross removal of the tumor; however, part of the tumor remained in the nasal cavity (Fig. 5).

The histological examination revealed that the tumor was composed of small round cells with uniform nuclei 
and scant cytoplasm (Fig. 6). Immunohistochemically, the cells were positive for epithelial markers, including cytokeratin (CK)8, CK18 and cytokeratin A (endo A), and for neuroendocrine differentiation markers, including synaptophysin (Syn), chromogranin A (ChrA), and neuron-specific enolase, but negative for carcinoembryonic antigen, epithelial membrane antigen, thyroid transcription factor 1 and ACTH (Figs. 7 and 8). The Ki-67 labeling index was $<1 \%$. Electron microscopy revealed the presence of occasional neuroendocrine granules (NEGs) (Fig. 9). These findings suggested low-grade small-cell NET.

The postoperative course was relatively uneventful. Abdominal CT scans revealed bilateral regression of the adrenal gland hyperplasia and the biochemical results revealed a potassium level of $4.25 \mathrm{mmol} / \mathrm{l}$, FBG level of 6.2-6.5 mmol/1, basal ACTH level of $1.42 \mathrm{pmol} / \mathrm{l}$ and 6 a.m. serum cortisol level of $272 \mathrm{nmol} / \mathrm{l}$.

\section{Discussion}

NETs originate from amine precursor uptake and decarboxylation cells, which are referred to as the diffuse neuroendocrine system (1-3), whereas intracranial origin is rare (0.74\%) (4). To the best of our knowledge, this is the first case of primary intracranial NET, immunonegative for $\mathrm{ACTH}$, resulting in Cushing's syndrome. In biochemical measurements, ChrA may be used as a tumor marker, as it increases in $70-90 \%$ of patients with NETs (5). On histological examination, the tumor is composed of large cells with uniform nuclei, scant cytoplasm, basophil granules and irregular mitoses (6). ChrA and Syn are selected as common immunohistochemical markers, which are sensitive and non-specific (7). According to the recent literature, testing for peptide hormones and bioamines is no longer recommended, as no correlation has been confirmed between hormone levels and clinical presentation, and the level of hormone is not a valuable prognostic indicator (8). The presence of intracytoplasmic NEGs on electron micrographs, whose compact cores show high electron density, is of value in the differential diagnosis.

Immunohistochemistry is a significant differential approach to the diagnosis of the neuroendocrine component, epithelial component and detection of neuropeptides. In this case, the tumor had to be differentiated from esthesioneuroblastoma and neuroendocrine carcinoma of the nasal cavity and paranasal sinuses. Klimstra (6) reported that esthesioneuroblstoma was composed of neuroblasts with uniform circular nuclei and longitudinal cerebromedullary tubes in the dendrites. NETs mainly originate from the epithelium, lacking a neural component. Immunohistochemistry of neuroendocrine carcinoma of the nasal cavity and paranasal sinuses is similar to that of the intracranial counterpart, but the Ki-67 labeling index is often $>3 \%$. As determined by imaging in this case, the bulk of tumor situated intracranially, with the skull base limiting tumor growth.

The NEGs were rare and immunohistochemistry was negative for ACTH, contradicting the preoperative changes in the serum ACTH level. The main reasons were as follows: i) ACTH consists of 39 amino acids, and is divided into the bioactive part, located in the first 24 amino acid residues, and the immunoactive part, located between the 22nd and

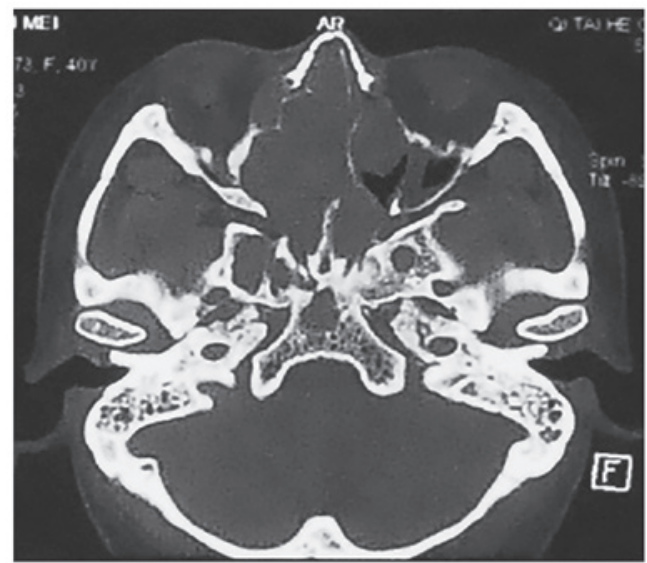

Figure 1. Bone computed tomography scans showing the neoplasm eroding the bones of the sphenoid and ethmoid sinuses bilaterally.

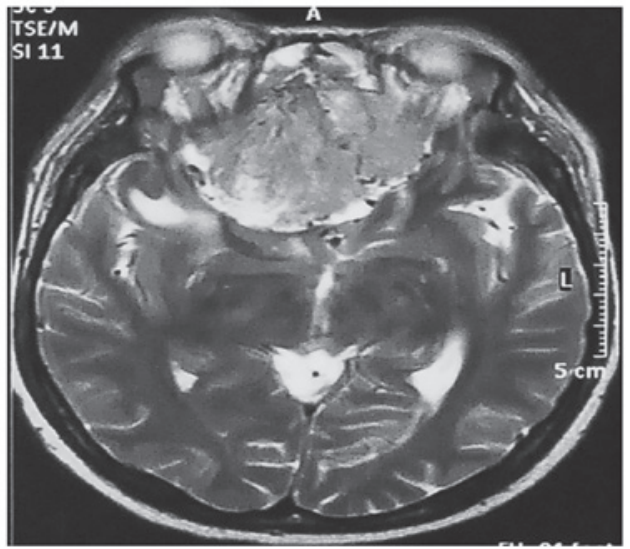

Figure 2. Preoperative axial magnetic resonance imaging scan demonstrating the contrast-enhanced tumor located in anterior cranial fossa.

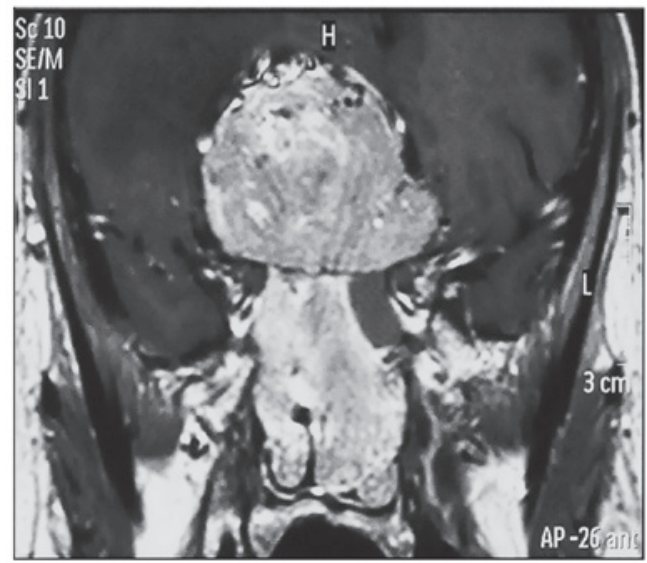

Figure 3. Preoperative coronary magnetic resonance imaging scan demonstrating the tumor infiltrating the nasal cavity.

39th amino acid residues (9). Thus, the antibody used in the Pathology Department of our institute may not recognize the secreted hormone. ii) Ectopic tumors may secrete ACTH or an ACTH analogue and a simple anti-ACTH antibody is insufficient for detecting ectopic secretion. iii) Some lesions may secrete ACTH and corticotropin-releasing hormone (CRH) 


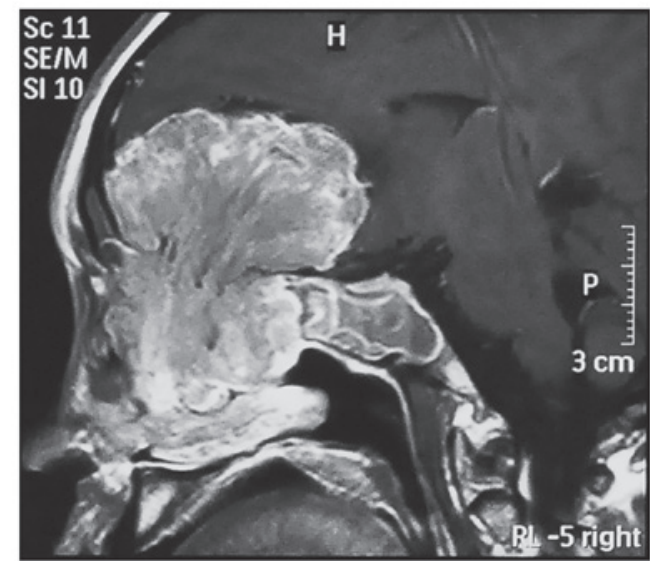

Figure 4. Preoperative sagittal magnetic resonance imaging scan demonstrating striped flow-empty activity on the superior tumor border.

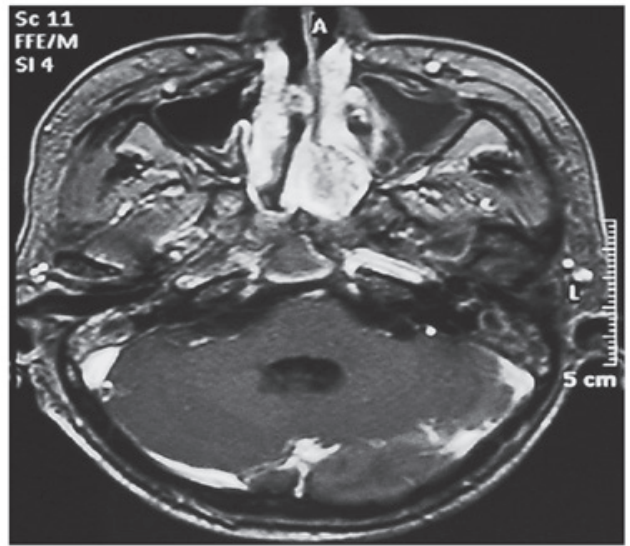

Figure 5. Postoperative magnetic resonance imaging scan (1 week) showing part of the lesion remaining in the nasal cavity.

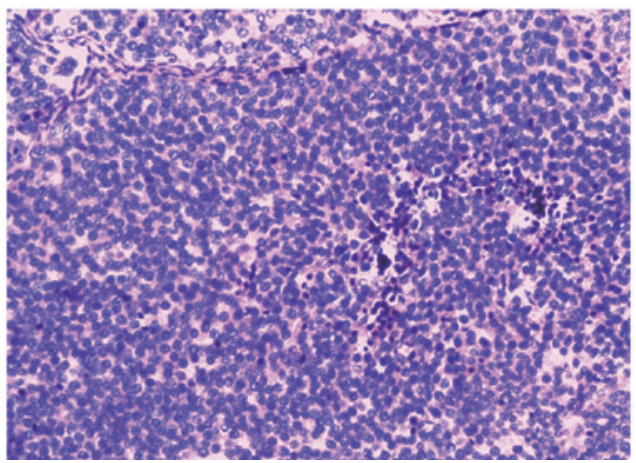

Figure 6. Histological findings of the surgically obtained specimen showing the tumor was composed of small round cells with uniform nuclei and scant cytoplasm (hematoxylin and eosin staining; magnification, $\mathrm{x} 100$ ).

simultaneously (10). Moreover, Al-Gahtany et al (11) reported three cases of ectopic CRH tumors, with pituitary hyperplasia leading to high corticotrophin hyperlipidemia; they also reported that, postoperatively, the ACTH level immediately returned to normal if the tumor produced $\mathrm{ACTH}$, but it decreased gradually if the tumor produced CRH. This patient's postoperative ACTH level returned to normal after 16 days. iv) The tumor had been present for 6 years, but

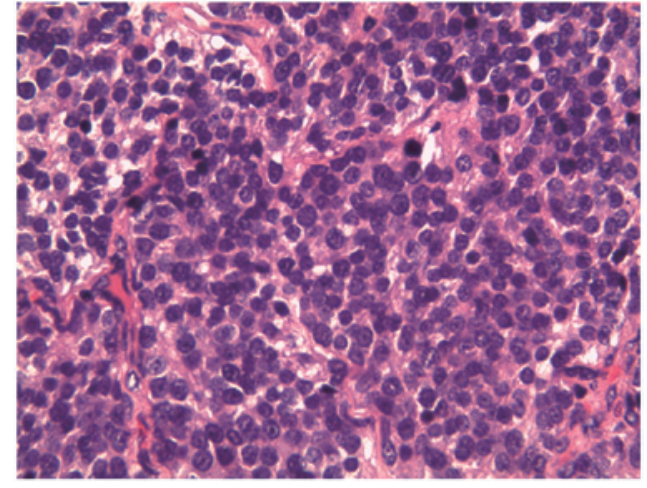

Figure 7. Immunohistochemically, the tumor cells exhibited diffuse cytoplasmic reactivity for $\mathrm{CD} 34$ (magnification, $\mathrm{x} 400$ ).

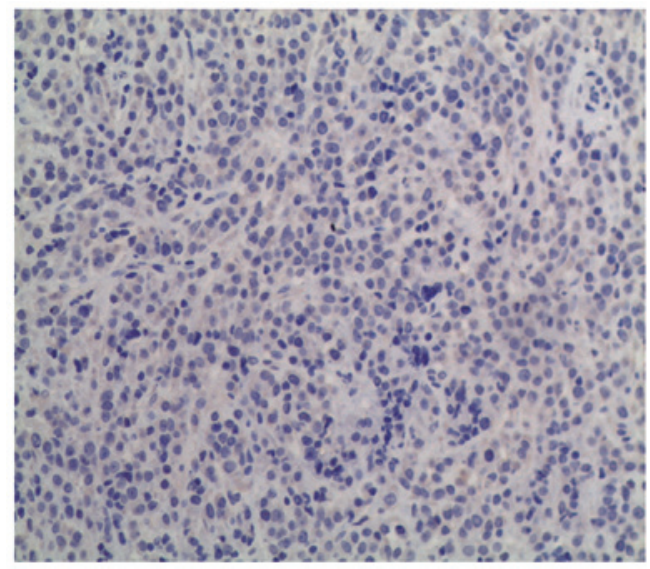

Figure 8 . The tumor cells were immunohistochemically positive for synaptophysin (magnification, x100).

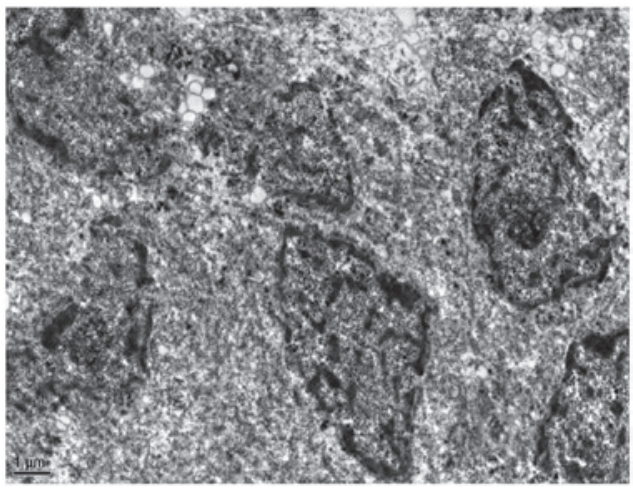

Figure 9. Electron microscopy revealed occasional cytoplasmic neuroendocrine granules (magnification, $\mathrm{x} 10,000$ ).

Cushing's syndrome had been present for 2 months. During a follow-up of $\sim 6$ months due to the residual tumor in the nasal cavity, the patient did not present with signs or symptoms of hypercortisolism. The reason may be that the ectopic hormone secretion by the residual NET was not sufficient to cause typical Cushing's syndrome.

The optimal treatment of NETs is surgical resection. Patients who receive comprehensive treatment, including completion of chemoradiotherapy, are expected to have longer 
tumor-free survival, compared with those receiving surgery alone (10). Drugs focusing on the epithelial component may also be selected.

Anterior skull base reconstruction was performed during surgery in this case. It was previously suggested that bony reconstruction should be performed when the deficit is $>3 \mathrm{~cm}$ (12). The 'sandwich' technique is currently used, involving tight suturing of the galea aponeurotica or temporalis fascia and the skull base dura, bony rehabilitation and covering with periosteum.

In summary, to the best of our knowledge, this is the first case of primary intracranial NET with Cushing's syndrome. The tumor was immunonegative for $\mathrm{ACTH}$ and may have displayed ectopic secretion of $\mathrm{CRH}$, or an ACTH analogue. The 'sandwich' skull base reconstruction technique was performed, using the quadriceps femoris, plastic skull flaps and periosteum, following tumor removal.

\section{References}

1. Godwin JD II: Carcinoid tumors. A analysis of 2837 cases. Cancer 36: 560-569, 1975.

2. Volante M, Rindi G and Papotti M: The grey zone between pure (neuro)endocrine and non-(neuro)endocrine tumors: A comment on concepts and classification of mixed exocrine-endocrine neoplasms. Virchows Arch 449: 499-506, 2006.

3. Shi J, Xu Z, Wang ZD, et al: Pathological category and diagnosis of neuroendocrine tumor in non-neuroendocrine system. J Clin Exp Pathol 25: 548-550, 2009 (In Chinese).
4. Faggiano A, Mansueto G, Ferolla P, Milone F, del Basso de Caro ML, Lombardi G, Colao A and De Rosa G: Diagnosis and prognostic implication of the World Health Organization classification of neuroendocrine tumors. J Endocrinol Invest 31: 216-223, 2008

5. Lambert SW, Hofland LJ and Nobels FR: Neuroendocrine tumor markers. Front Neuroendocrinol 22: 309-339, 2001.

6. Klimstra DS: Pathology reporting of neuroendocrine tumors: Essential elements for accurate diagnosis, classification and staging. Semin Oncol 40: 23-36, 2013.

7. Travis WD, Rush W, Flieder DB, Falk R, Fleming MV, Gal AA and Koss MN: Survival analysis of 200 pulmonary neuroendocrine tumors with classification of criteria for atypical carcinoid and its separation from typical carcinoid. Am J Surg Pathol 22: 934-944, 1998 .

8. Hochwald SN, Zee S, Conlon KC, Colleoni R, Louie O, Brennan MF and Klimstra DS: Prognostic factors in pancreatic endocrine neoplasms: An analysis of 136 cases with a proposal for low-grade and intermediate-grade groups. J Clin Oncol 20: 2633-2642, 2002.

9. Smith EM, Galin FS, LeBoeuf RD, Coppenhaver DH, Harbour DV and Blalock JE: Nucleotide and amino acid sequence of lymphocyte-derived corticotropin: Endotoxin induction of a truncated peptide. Pro Natl Sci USA 87: 1057-1064, 1990.

10. Kolesnikova GS, Lapshina AM, Voronkova IA, Marova EI, Arapova SD, Goncharov NP and Dedov II: Comparative analysis of clinical, hormonal and morphological studies in patients with neuroendocrine ACTH-producing tumors. Int J Endocrinol 2013: 659232, 2013

11. Al-Gahtany M, Horvath E and Kovacs K: Pituitary hyperplasia. Hormones (Athens) 2: 149-158, 2003.

12. Liu H, Zhang Q and Yang Z: Skull base reconstruction and rehabilitation. J Clin Otorhinolaryngol 18: 755-757, 2003 (In Chinese). 